REVISTA CIENCIAS BIOMÉDICAS

HISTORIA DE LA MEDICINA EN CARTAGENA

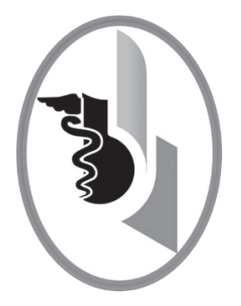

\title{
RAFAEL ALVEAR TERÁN: EL ÚLTIMO EXPONENTE DEL MÉTODO ANATOMOCLÍNICO EN LA UNIVERSIDAD DE CARTAGENA*
}

\author{
RAFAEL ALVEAR TERÁN: THE LAST EXPONENT OF THE \\ ANATOMO-CLINICAL METHOD IN THE UNIVERSIDAD DE \\ CARTAGENA \\ Mercado-Lara María Fernanda ${ }^{1}$ \\ Monterrosa-Castro Álvaro² \\ Correspondencia: mafemercado0898@gmail.com \\ Recibido para evaluación: marzo -25 - 2015. Aceptado para publicación: octubre - 20 - 2015.
}

\section{RESUMEN}

La anatomía humana fue el epicentro del saber, quehacer y la educación médica durante finales del siglo XVIII y comienzos del siglo XIX. A este movimiento proveniente de escuelas médicas de París se le denominó el método anatomoclínico y promulgaba la articulación de los signos y síntomas de una patología con los hallazgos o lesiones anatómicas. Lo anterior permitió el auge de los anatomistas y el desarrollo de didácticas en el aprendizaje de la medicina con la disección de cadáveres humanos en los anfiteatros. Investigación, educación y asistencia clínica se realizó por décadas bajo el amparo conceptual de la medicina anatomoclínica. Con el pasar de los años surgió la medicina de laboratorio y otras corrientes como la etiopatológica y la fisiopatológica, las cuales trajeron como consecuencia que el papel de la anatomía dentro de la enseñanza y la práctica clínica fuese modificado. La Facultad de Medicina de la Universidad de Cartagena-Colombia recibió influencia de la medicina anatomoclínica por parte de docentes que realizaron sus estudios médicos en escuelas francesas y por la lectura de libros en francés. Una saga de profesores anatomistas se pueden identificar, siendo el doctor Rafael Alvear Terán, el último docente de esa facultad médica, representante de la también llamada mentalidad anatomoclínica. Rev.cienc.biomed. 2015;6(2): 394-405

PALABRAS CLAVE

Historia de la medicina; Educación médica; Historiografía; Anatomía.

\section{SUMMARY}

Human anatomy was the focus of knowledge, pursuit and medical education in the late eighteenth century and early nineteenth century. This movement came from medical schools in Paris and was called the anatomo-clinical method; this method spread the articulation of the signs and symptoms in pathology findings or anatomical lesions. Also, this allowed the growth of anatomist and development of teaching learning in medicine with dissection of human cadavers in dissecting room. Investigation, education and clinical assistance were carried out for decades under the anatomo-clinical method.

* Un producto del Semillero de investigación Historiografía Médica Cartagenera (HISTORIMED).

2 Estudiante de Pregrado. Medicina. Semillero de Investigación Historiografía Médica Cartagenera (HISTORIMED). Facultad de Medicina. Universidad de Cartagena. Colombia.

3 Médico. Profesor titular. Semillero de Investigación Historiografía Médica Cartagenera (HISTORIMED). Facultad de Medicina. Universidad de Cartagena. Colombia. 
Laboratory medicine and others areas like physiopathology and etiopathology emerged with the passing of time, which brought as a consequence that the role of anatomy in teaching and clinical practice was modified. The Medicine department in the Universidad de Cartagena-Colombia was influenced by professors who study medicine in French schools and books of the same origin. Inside this medical department there were many anatomists but the last exponent of the anatomo-clinical method was Rafael Alvear Terán. Rev.cienc.biomed. 2015;6(2):394-405

\section{KEYWORDS}

History of medicine; Medical education; Historiography; Anatomy.

\section{INTRODUCCIÓN}

En la Edad Media existía poco interés en el papel que el organismo pudiese tener para explicar las condiciones de salud o enfermedad, estas dependían de la posesión de espíritus maléficos que invadían el cuerpo del enfermo. Muy pocos libros sobre anatomía se han encontrado de esas épocas, por ello son casi exclusivos los textos realizados por Galeno. Fue Mondino di Luzzi (1276-1326) uno de los primeros en luchar contra la oposición que realizaba la iglesia para la práctica de disecciones anatómicas. Los cientos de dibujos anatómicos realizados muchos años después por Leonardo Da Vinci (14521519) son importantes y han sido ampliamente reconocidos, en ellos se identifica la profundidad de las observaciones anatómicas. Posteriormente, empezaría a definirse la importancia que la anatomía debía tener dentro del estudio médico y en la explicación de diferentes fenómenos mórbidos.

En las postrimerías del siglo XVIII e inicios del siguiente se presentó el nacimiento de una corriente conceptual que fundamentó a la anatomía para el ejercicio, el aprendizaje y la enseñanza de la medicina. La estructura y el componente anatómico se definieron como piezas fundamentales y se estableció su relación con el cuadro clínico. Se sentaron las bases para considerar la posibilidad de establecer un diagnóstico patológico a partir del elemento puramente anatómico. Esta propuesta que permitió establecer un conocimiento científico basado en la observación y en la objetividad pasaría a ser identificada como el método anatomoclínico. Al respecto, Jean Charles Sournia señaló que esa mirada médica que relaciona el cuerpo vivo con el cadáver podía ser denominada "conversión anatomoclínica".
Dicho método vino a ser la correlación entre los hallazgos anatómicos con los síntomas o alteraciones funcionales que correspondían a una entidad nosológica. El método anatomoclínico hizo posible relacionar lesiones específicas orgánicas con cuadros clínicos complejos. Alrededor de la lesión anatómica fueron organizadas las manifestaciones y los signos que se encontraban al realizar evaluación o exploración médica. La experiencia clínica comenzó a abrirse un nuevo espacio en lo material de la morfología corporal, en la medida en que se identificaban estructuras orgánicas normales y patológicas. Los órganos y los tejidos somáticos se convirtieron en los elementos centrales del fenómeno patológico y en el acontecimiento mórbido para la explicación de las enfermedades.

Marie-Francois-Xavier Bichat (1771-1802) y René Théophile Hyacinthe Laënnec (17811826) son considerados como los fundadores del método anatomoclínico, por la amplitud y explicitud con la cual sentaron las bases para un cambio que modificó no solamente la manera de denominar a las alteraciones, sino comprenderlas y abordarlas desde el punto de vista terapéutico.

El conocimiento preciso y minucioso de la topografía y estructuras anatómicas debía ser adquirido dentro de los programas de formación médica. Fue fundamental el conocimiento detallado del organismo y sus componentes anatómicos para poder visibilizar las alteraciones que producían las enfermedades. Ello era coherente con las sentencias de Bichat, quien solicitaba: "Abrir los cadáveres para poder obtener el conocimiento de la ciencia médica".

La observación detallada y organizada permitía disipar la carencia de conocimiento. 
Dentro del concepto anatomoclínico la enfermedad dejaba de ser un componente lejano o abstracto, posiblemente mítico, mágico o religioso para convertirse en un elemento concreto, medible y con la posibilidad de ser intervenido. Eso motivó la aparición de importantes profesionales de la nueva concepción médica, quienes se definieron como anatomistas, expresión que fue ampliamente utilizada en el siglo anterior para identificar a la persona que se dedicaba al estudio de la anatomía, experto en esa ciencia o profesor de la misma. Algunos de los más representativos dentro de la historia médica universal aparecen a continuación en la Tabla No 1.

\begin{tabular}{|l|l|}
\hline \multicolumn{2}{|c|}{ TABLA No 1. } \\
ANATOMISTAS CON IMPORTANTES APORTES \\
A LA MEDICINA UNIVERSAL
\end{tabular}

\begin{tabular}{|c|c|}
\hline $\begin{array}{l}\text { Bowman Sir } \\
\text { William } \\
(1816-1892)\end{array}$ & $\begin{array}{l}\text { - Médico y anatomista } \\
\text { inglés. } \\
\text { - Describió la cápsula de } \\
\text { Bowman. }\end{array}$ \\
\hline $\begin{array}{l}\text { Broca Paul } \\
(1824-1880)\end{array}$ & $\begin{array}{l}\text { - Cirujano y anatomista } \\
\text { francés. } \\
\text { - Describió el área y la } \\
\text { afasia de Broca. }\end{array}$ \\
\hline $\begin{array}{l}\text { Brunner Johann } \\
\text { Conrad } \\
(1635-1895)\end{array}$ & $\begin{array}{l}\text { - } \text { Anatomista suizo. } \\
\text { - Describió las glándulas de } \\
\text { Brunner. } \\
\end{array}$ \\
\hline $\begin{array}{l}\text { De Graaf, Regnier } \\
(1641-1673)\end{array}$ & $\begin{array}{l}\text { - } \text { Médico y anatomista } \\
\text { neerlandés. } \\
\text { Describió los túbulos } \\
\text { seminíferos. } \\
\text { - Describió los conductos } \\
\text { deferentes. } \\
\text { - Propuso el } \\
\text { funcionamiento de las } \\
\text { trompas de falopio. } \\
\text { Identificó y explicó el } \\
\text { folículo de De Graaf. }\end{array}$ \\
\hline $\begin{array}{l}\text { Eustaquio } \\
\text { Bartolomé } \\
(1520-1574)\end{array}$ & $\begin{array}{l}\text { - Anatomista y médico } \\
\text { italiano. } \\
\text { - Describió las glándulas } \\
\text { suprarrenales humanas. } \\
\text { - Describió la trompa de } \\
\text { Eustaquio. }\end{array}$ \\
\hline $\begin{array}{l}\text { Fabricius } \\
\text { Hieronymus } \\
(1537-1619)\end{array}$ & $\begin{array}{l}\text { - } \quad \text { Anatomista italiano. } \\
\text { - } \quad \text { Explicó la formación del } \\
\text { feto. } \\
\text { - Describió la estructura } \\
\text { esofágica, estomacal e } \\
\text { intestinal. } \\
\text { - Describió los pliegues } \\
\text { membranosos en el } \\
\text { interior de las venas. }\end{array}$ \\
\hline $\begin{array}{l}\text { Gimbernat Antonio } \\
\text { de } \\
(1734-1816)\end{array}$ & $\begin{array}{l}\text { - } \text { Cirujano y anatomista } \\
\text { español. } \\
\text { Describió el canal } \\
\text { inguinal. } \\
\text { - Propuso una técnica de } \\
\text { reparación para la hernia } \\
\text { crural. }\end{array}$ \\
\hline $\begin{array}{l}\text { Grüber Wenzel } \\
\text { Leopold } \\
(1814-1890)\end{array}$ & $\begin{array}{l}\text { - Anatomista ruso. } \\
\text { - Describió la hernia de } \\
\text { Grüber. } \\
\text { - } \\
\text { Gescribió la fosa de } \\
\text { Grüber-Landzert. }\end{array}$ \\
\hline $\begin{array}{l}\text { Hoffmann Morizt } \\
(1622-1698)\end{array}$ & $\begin{array}{l}\text { - Anatomista alemán. } \\
\text { - Describió el conducto de } \\
\text { Hoffman. }\end{array}$ \\
\hline $\begin{array}{l}\text { Horner William } \\
\text { Edmund } \\
(1793-1853)\end{array}$ & $\begin{array}{l}\text { - Anatomista } \\
\text { norteamericano. } \\
\text { - Describió el músculo de } \\
\text { Horner. }\end{array}$ \\
\hline
\end{tabular}


SIGUE

\begin{tabular}{|c|c|}
\hline $\begin{array}{l}\text { Huber Jean Jacques } \\
(1707-1778)\end{array}$ & $\begin{array}{l}\text { - Anatomista suizo. } \\
\text { - Describió el ganglio de } \\
\text { Huber. }\end{array}$ \\
\hline $\begin{array}{l}\text { Hunter John } \\
(1728-1793)\end{array}$ & $\begin{array}{l}\text { - Anatomista y cirujano } \\
\text { inglés. } \\
\text { - Describió la glositis de } \\
\text { Hunter. }\end{array}$ \\
\hline \begin{tabular}{|l} 
Huschke Emil \\
$(1797-1858)$
\end{tabular} & $\begin{array}{l}\text { - } \text { Anatomista alemán. } \\
\text { - } \text { Hescribió el foramen de } \\
\text { - Deschke. } \\
\text { Huschibió la válvula de } \\
\text { Husche }\end{array}$ \\
\hline $\begin{array}{l}\text { Jacobson Ludwig } \\
\text { Levin } \\
(1783-1843)\end{array}$ & $\begin{array}{l}\text { - Anatomista dinamarqués. } \\
\text { - Describió el órgano de } \\
\text { Jacobson. } \\
\text { - Describió el conducto de } \\
\text { Jacobson. }\end{array}$ \\
\hline \begin{tabular}{|l} 
Malpighi Marcello \\
$(1628-1694)$
\end{tabular} & $\begin{array}{l}\text { - } \quad \text { Médico y anatomista } \\
\text { italiano. } \\
\text { - } \quad \text { hundador de la anatomía } \\
\text { - } \quad \text { Describió los corpúsculos } \\
\text { de Malpighi. } \\
\text { - Completó los trabajos } \\
\text { sobre la circulación } \\
\text { sanguínea de Harvey. } \\
\end{array}$ \\
\hline $\begin{array}{l}\text { Meckel Johann } \\
\text { Friedrich } \\
(1781-1833) \\
\end{array}$ & $\begin{array}{l}\text { - Anatomista alemán. } \\
\text { - Describió el ganglio de } \\
\text { Meckel. }\end{array}$ \\
\hline $\begin{array}{l}\text { Meibom Heinrich } \\
(1638-1700)\end{array}$ & $\begin{array}{l}\text { - } \text { Anatomista alemán. } \\
\text { - Describió las glándulas de } \\
\text { Meibomius. }\end{array}$ \\
\hline $\begin{array}{l}\text { Morgagni Giovanni } \\
\text { Battista } \\
(1682-1771)\end{array}$ & $\begin{array}{l}\text { - } \quad \text { Anatomista italiano. } \\
\text { - } \text { pundador de la anatomía } \\
\text { patoloica. } \\
\text { Describió el infantilismo } \\
\text { sexual. }\end{array}$ \\
\hline $\begin{array}{l}\text { Riolan Jean } \\
(1577-1657)\end{array}$ & $\begin{array}{l}\text { - Médico y anatomista } \\
\text { francés. } \\
\text { - Describió el ramillete de } \\
\text { Riolan. } \\
\text { - Describió los fascículos } \\
\text { internos del orbicular de } \\
\text { los párpados. }\end{array}$ \\
\hline $\begin{array}{l}\text { Rivinus Augustus } \\
\text { Quirinus } \\
(1652-1723)\end{array}$ & $\begin{array}{l}\text { - Médico y anatomista } \\
\text { alemán. } \\
\text { Describió el conducto de } \\
\text { Rivinus. }\end{array}$ \\
\hline $\begin{array}{l}\text { Sylvius François de } \\
\text { la Boe } \\
(1614-1672)\end{array}$ & $\begin{array}{l}\text { - Anatomista alemán. } \\
\text { - Describió la cisura de } \\
\text { Silvio. }\end{array}$ \\
\hline $\begin{array}{l}\text { Varolio Constanzo } \\
(1542-1575)\end{array}$ & $\begin{array}{l}\text { - Anatomista y cirujano } \\
\text { italiano. } \\
\text { - Describió el puente de } \\
\text { Varolio. }\end{array}$ \\
\hline
\end{tabular}

\begin{tabular}{|c|c|}
\hline $\begin{array}{l}\text { Wharton Thomas } \\
(1614-1673)\end{array}$ & $\begin{array}{l}\text { - Médico y anatomista } \\
\text { inglés. } \\
\text { - Describió el conducto de } \\
\text { Wharton. }\end{array}$ \\
\hline $\begin{array}{c}\text { Willis Thomas } \\
(1621-1675)\end{array}$ & $\begin{array}{l}\text { - Anatomista y médico } \\
\text { inglés. } \\
\text { - Describió el polígono de } \\
\text { Willis. }\end{array}$ \\
\hline $\begin{array}{l}\text { Wolff Kaspar } \\
\text { Friedrich } \\
(1733-1794)\end{array}$ & $\begin{array}{l}\text { - Anatomista y fisiólogo } \\
\text { francés. } \\
\text { - Pionero de la } \\
\text { embriología. } \\
\text { - Describió el cuerpo de } \\
\text { Wolff. }\end{array}$ \\
\hline $\begin{array}{l}\text { Zuckerkandl Emil } \\
(1849-1910)\end{array}$ & $\begin{array}{l}\text { - Anatomista vienés. } \\
\text { - Describió la } \\
\text { circunvolución de } \\
\text { Zuckerkandl. } \\
\text { - Describió el órgano de } \\
\text { Zuckerkandl. }\end{array}$ \\
\hline
\end{tabular}

Con el método anatomoclínico se presentó sustancialmente un cambio, una ruptura de las posiciones médicas de muchos milenios frente a la presencia de la enfermedad. Entonces la medicina fue adquiriendo posturas que pueden considerarse científicas, que modificaron el imaginario colectivo tanto en la comunidad general como en el profesional médico particular.

El debate sobre la teorización y conceptualización del papel de la anatomía era interesante y candente mientras se identificaban estructuras anatómicas. Téophile de Bordeu (1722-1776) fue uno de los creadores del concepto de anatomía general, identificado como amplio estudioso del concepto "tejido orgánico" que Gabriel Falopio había acuñado en el siglo XVI (Falopio fue el sucesor de Andrea Vesalio en su cátedra de anatomía). Bordeu consideró al tejido como una estructura continua y fundamental dentro de la funcionabilidad del organismo y estuvo en contra del parecer de Morgagni (1682-1771) que establecía un accionar de los órganos como elementos aislados.

\section{EL IMPACTO EN CARTAGENA}

La educación médica cartagenera nació cuando la medicina anatomoclínica aún estaba en la cumbre como referente de modelo educativo médico. La aplicación y desarrollo del método anatomoclínico hizo que la medicina 
francesa se colocara a la cabeza de la formación médica mundial en los inicios del siglo XIX, llegando a irradiar al continente europeo y en virtud de las modificaciones geopolíticas al ámbito hispanoamericano. Con el componente educativo y asistencial el modelo anatomoclínico se extendió hacia Latinoamérica e influyó marcadamente en los programas de educación médica que se impartieron hasta el final de la primera mitad del siglo XX.

Por más de cien años los modelos curriculares, los planes de estudio, la teorización y el desempeño profesional de los médicos en América Latina en gran parte fueron de corte francés y con posturas enmarcadas en el método anatomoclínico. Ackerknecht señaló que París se convirtió en la "Meca de los estudiantes venidos de todos los rincones del planeta". El componente de investigación y exploración anatómica con fines de búsqueda y génesis de nuevo conocimiento no fue transferido ni desarrollado. Solo un plan transmisionista y de aplicación de lo ya conocido fue la realidad latinoamericana en cuanto al método anatomoclínico.

A mediados del siglo XVIII se realizaron los primeros intentos de educación médica en
Colombia, todos fallidos, ya que estaban caracterizados por el empirismo y la ausencia de ordenamiento educativo al interior de un espacio político convulso. Fue solo hasta 1802 cuando se inició el plan de estudios propuesto por José Celestino Mutis (17321808) que incluyó la enseñanza de la anatomía desde el primer año, acompañada de prácticas bajo supervisión. La enseñanza de la anatomía se realizó con aportes pedagógicos donde el estudiante aprendía de memoria conceptos teóricos que complementaba con la explicación de dibujos o figuras anatómicas y con las prácticas de disección de cadáveres en el anfiteatro.

Muchos años después, en el año 1891, fue tomada una fotografía que muestra la disección anatómica humana en ámbitos de enseñanza universitaria, tomada a profesores y estudiantes de la Facultad de Medicina de la Universidad de Cartagena (Figura No.1). En esa época el país, al igual que en casi toda Latinoamérica, tanto la enseñanza de la anatomía en particular como la de medicina en general, eran de tipo memorístico y casi exclusivamente magistral. Elementos que son propios de la medicina francesa.

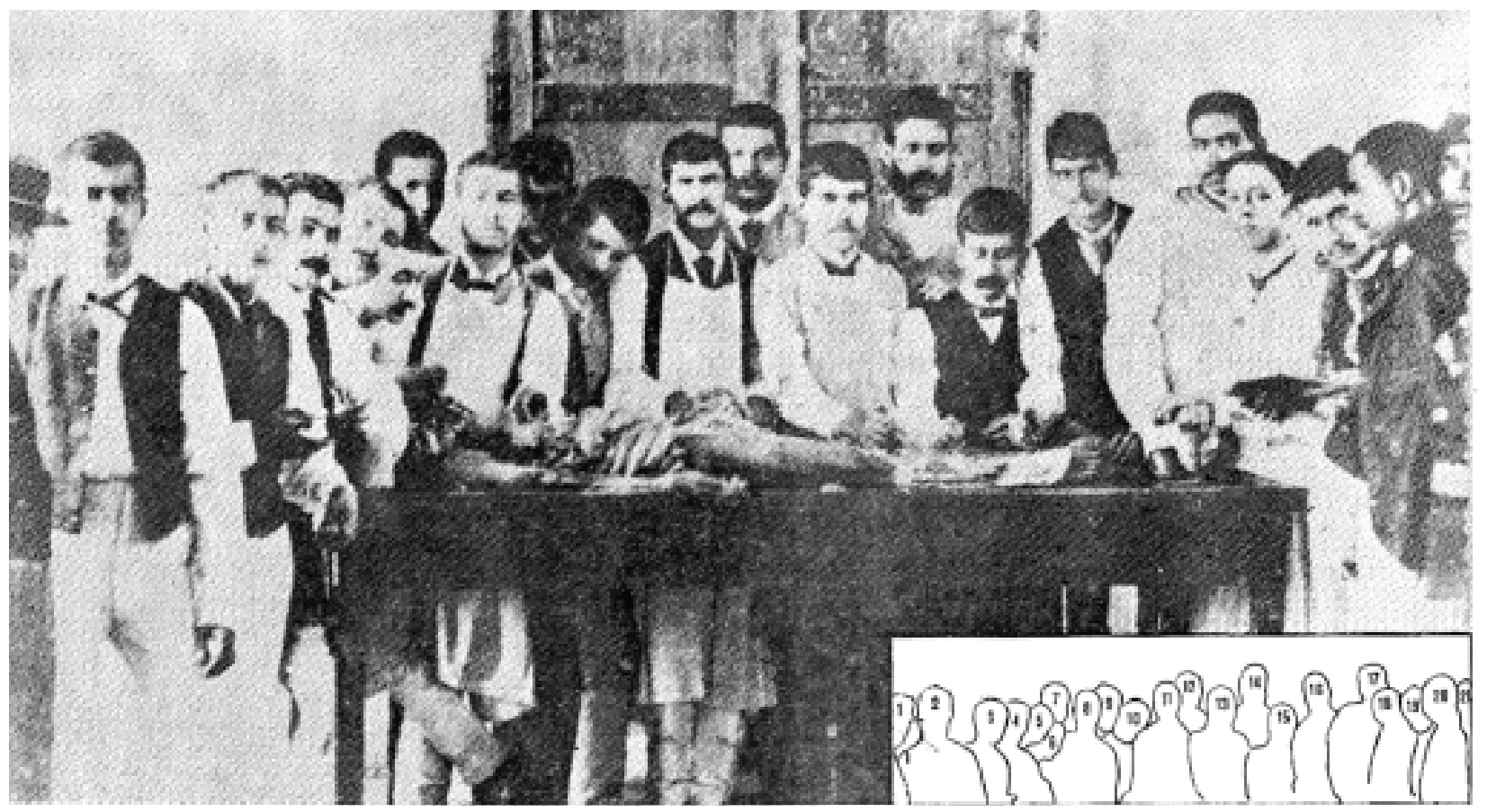

FIGURA N ${ }^{\circ}$. Curso de Anatomía de 1891 en el Anfiteatro de la Facultad de Medicina de la Universidad de Cartagena. Entre los alumnos se pueden distinguir a Francisco Sotomayor S. (8), Miguel R. Méndez (11), Teofrasto Tatis (13), Miguel A. Lengua (16) y Rafael Calvo (18). 
Diversos argumentos explican las razones por las cuales el método anatomoclínico y toda la medicina francesa encontraron ambiente propicio en Colombia para su fuerte presencia en todos los programas médicos que se impartían en el territorio nacional: [A] las fáciles y frecuentes relaciones con el pueblo francés, [B] la similitud con el idioma, [C] la relativa disponibilidad de libros como materiales de lectura que facilitaban la enseñanza y el aprendizaje, [D] la presencia de relaciones comerciales y [E] las cercanías políticas y religiosas.

Por su parte, en el año 1827 fue creada la actual Universidad de Cartagena con el nombre de Universidad del Magdalena e Istmo en el Caribe colombiano. Simultáneamente fue creada la Facultad de Medicina, la se desarrolló en forma poco formal e inconstante en las primeras dos décadas y más estructuradamente desde los años cincuenta de ese siglo gracias al aporte académico y administrativo que realizaron los doctores Rafael Calvo Lamadrid y Vicente A. García, quienes habían tenido formación médica en Francia. Desde ese momento hasta la mitad del siglo XX, dicha escuela médica tuvo un corte notoriamente influenciado por la medicina francesa, tanto en la organización docente como en la praxis médica que adelantaban sus egresados. Con accionar docentes y proyectos académicos solamente profesionalizantes generaron un favorable impacto social en la comunidad. La innovación y la investigación de nuevo conocimiento fueron terrenos distanciados, por una u otra razón, para el cuerpo profesoral y para los discentes en formación. Lo referente a investigación se circunscribió a describir las observaciones y experiencias vividas, o a reescribir lo publicado. Ello fue igual a lo que sucedía en la mayoría de las instituciones de educación médica a nivel latinoamericano.

Por ello los anatomistas latinoamericanos fueron los profesores que se destacaron en la enseñanza de los conceptos anatómicos. En ese tiempo en la Facultad de Medicina de la Universidad de Cartagena, la anatomía se enseñaba rigurosamente y a profundidad durante los años de estudios médicos, era una asignatura transversal, tal como se conoce hoy. La disección anatómica en cadá- veres era fuente importante de conocimiento médico y permitía generar una visión amplia sobre la cual se sentaba el proceder profesional y la toma de decisiones terapéuticas. Las demostraciones realizadas por los profesores facilitaban la extrapolación de los conceptos anatómicos del cadáver al ámbito clínico. Como era común en ese período, la enseñanza médica encajaba en un paradigma pedagógico tradicional donde compartir el conocimiento ya elaborado era lo esencial. De los libros importados especialmente desde Francia se tomaba la actualización y se transmitía acompañada de la experiencia personal del docente.

El docente era el erudito en cada una de las patologías y así era identificado. Ellos presentaban toda la teoría y el estudiante escuchaba, memorizaba y relacionaba los signos y síntomas con los órganos o las estructuras anatómicas, era común considerar a la anatomía como la principal área del conocimiento, alrededor de ella giraban todos los demás temas clínicos o médicos.

Fue el doctor Vicente A. García el propiciador e iniciador de los estudios anatómicos en la Facultad de Medicina de la Universidad de Cartagena quien cumplió con su cometido bajo condiciones incipientes y hostiles. Rafael Calvo Lamadrid lo sucedió en su gestión y por muchos años fue el profesor encargado de la enseñanza práctica de la anatomía. Una placa de mármol colocada en el anfiteatro del Hospital Universitario Santa Clara hizo justicia, principalmente, a su trayectoria como anatomista, a su dedicación como docente y al valioso aporte que entregó a la Universidad.

Rafael Calvo Castaño, hijo de Rafael Calvo Lamadrid, fue quien trajo a inicios del siglo XX de los Estados Unidos la fórmula Rawson para inyectar cadáveres en la arteria femoral y carótida con fines de conservación. Teofrasto A. Tatis, en esa misma época gestionó por vez primera la consecución de piezas anatómicas y fue un gran impulsador para el crecimiento del anfiteatro anatómico, mejorando las condiciones locativas. Teofrasto A. Tatis está en la fotografía tomada en el anfiteatro de la Facultad de Medicina de la Universidad de Cartagena (Figura $N^{\circ} 1$ ). 
Los dos profesores señalados realizaron aportes sensibles para que se diese este importante progreso en la enseñanza de la anatomía. Este período es especialmente recordado por el profesor Horacio Zabaleta Jaspe en su libro "Réquiem por un viejo hospital", donde señaló: "Los estudiantes o practicantes de medicina leían en libros franceses o alemanes a la luz de titilantes velas, de tenebrosos candiles o de ahumadas pajuelas. Los estudios de la anatomía se realizaban en un anfiteatro en el trasfondo del Hospital Santa Clara. Un cadáver duraba dos o más años, el aire salino del mar los cubría de verdín, de moho a manera de penicilina y hasta los oxidaba junto a las mesas de hierro del local, por la humedad permanente".

Varios profesores de la Facultad de Medicina de la Universidad de Cartagena fueron denominados anatomistas y se destacaron por el amplio conocimiento de la anatomía humana, la experticia que tenían en la identificación de las diferentes estructuras, variedades, relaciones y correlaciones, en general, en todo lo que hacía parte de la anatomía topográfica o descriptiva. Ellos fueron exigentes y sumamente rígidos en su enseñanza, lo cual era coherente con las corrientes europeas. Poseer amplios y profundos conocimientos anatómicos, era el antecedente o requisito central e imprescindible para el abordaje clínico.

En la Facultad de Medicina existió por años el cargo de Disector anatómico, el cual alcanzaban por concurso los estudiantes de medicina de últimos años. Los disectores anatómicos cumplieron importante labor como auxiliares de docencia en el anfiteatro. La denominación cambiaría posteriormente a monitores de anatomía macroscópica y luego a monitores de morfología, hasta que desaparecieron con el fin del siglo XX.

La cátedra de anatomía humana en dicha facultad fue adelantada entre otros, por los doctores: Vicente A. García, Rafael Calvo Lamadrid, su hijo Rafael Calvo Castaño, Manuel Pájaro Herrera, Lascario Barbosa, Enrique Solano, José A. Caballero Lecler, Manuel Francisco Obregón Flórez y luego 1925 su hijo Francisco Obregón Jaraba, desde que regresó de París donde había sido alumno del anatomista A. Latarjet. El profesor Obregón Jaraba ejerció el cargo de jefe de anatomía y por tanto, fue el docente de la cátedra por cerca de treinta años.

Cabe destacar que el 29 de julio de 1931, el profesor de la Facultad de Medicina de París, André Latarjet (1877-1947), coautor con Leo Testut (1849-1925) del voluminoso tratado en cuatro tomos de anatomía humana conocido como Testut-Latarjet y utilizado por muchos años como libro básico de la enseñanza de la anatomía visitó la Universidad de Cartagena, el Hospital Universitario Santa Clara y el Lazareto de Caño del Oro. Vino junto con los profesores Paul Durand y Louis Tavernier y dejaron importantes recomendaciones para mejorar el funcionamiento educativo de la Facultad de Medicina.

Posterior a Obregón Jaraba, la cátedra anatómica fue encargada al doctor Carlos Manuel Esquivia Cortina, quien era preciso, claro y práctico en sus enseñanzas. Sus clases empezaban a las siete de la mañana cada dos días y las prácticas de disección eran diarias y se extendían por muchas horas. El texto de Leo Testut y André Latarjet, continuó siendo utilizado en la enseñanza de la anatomía.

A finales del año cincuenta se comenzó a utilizar el también voluminoso libro de Henry Rouviere (1876-1952), también de origen francés. Las exigencias de la enseñanza y aprendizaje de la anatomía se conservaban, aunque surgían libros Ilamados "compendios" que resumían al máximo las propuestas de los grandes tratados. Era común la resistencia de los docentes al estudio en estas pequeñas apuestas para aprender la anatomía.

El último exponente de la enseñanza anatómica exhaustiva y ampliamente detallada dentro de la Facultad de Medicina de la Universidad de Cartagena fue el doctor Rafael Alvear Terán, quien cumplió su labor docente entre los años cincuenta y setenta del siglo $X X$. Durante su práctica profesoral cumplió lo que se destilaba desde años atrás, enseñanza de una anatomía estática, pura y puntal, donde se exploraba los huesos desde la cabeza hasta los pies (dividida en partes y con elevada carga horaria). 
La anatomía primera se impartía en orden y por muchos meses e ininterrumpidamente detallando la osteología, el sistema muscular, el sistema arterial, venoso y linfático, los miembros superiores e inferiores, articulaciones, locomoción y funciones de relación. En la anatomía segunda: aparato digestivo, intestinal, sistema nervioso central y periférico, nervios, aparato genitourinario y órganos de la reproducción, corazón y los grandes vasos arteriales.

\section{RAFAEL ALVEAR TERÁN}

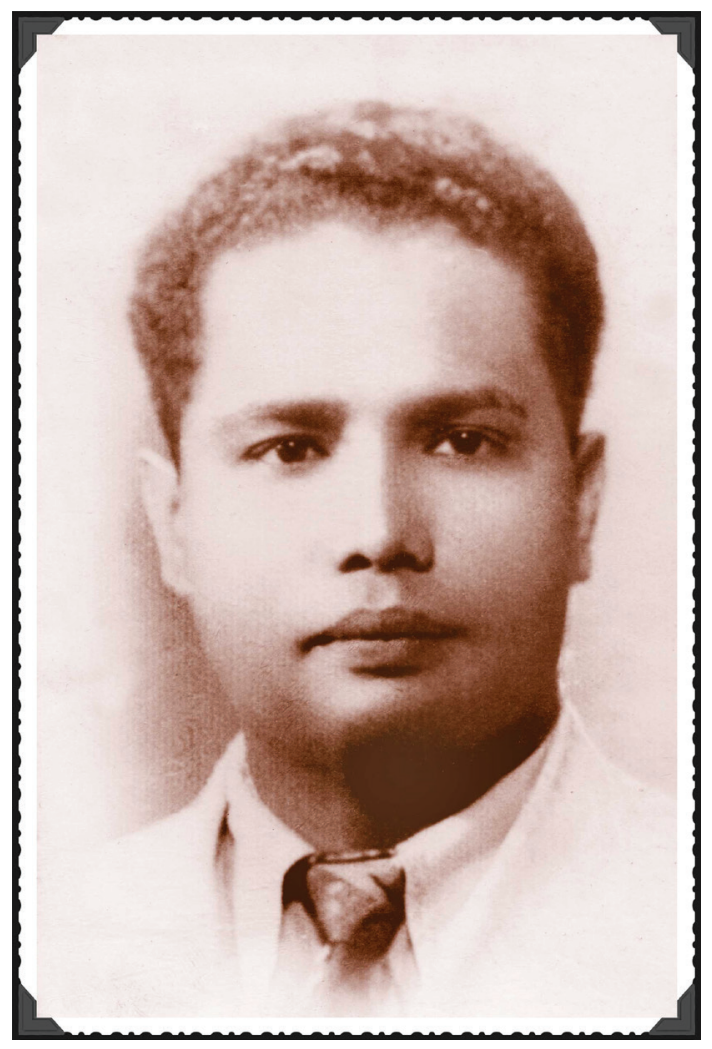

Rafael Alvear Terán nació el 9 de julio de 1907 en la población de Villa Rosa, corregimiento de Repelón, en el departamento del Atlántico, en el Caribe colombiano. En el año 1927 recibió su grado de bachiller y al año siguiente fue admitido como estudiante de la Facultad de Medicina de la Universidad de Cartagena.

Durante su etapa de estudiante universitario fue miembro activo y presidente del Centro Departamental de Estudiantes de Bolívar, en el cual puso todo su empeño y esfuerzo con el fin de defender los derechos de los estudian- tes y buscar mejoras en la calidad académica de la facultad. Fue disector anatómico de la Facultad de Medicina, cargo que equivale a las monitorías actuales, el cual alcanzó por medio de concurso. Además fue practicante de la consulta externa del Hospital Santa Clara, también alcanzado por concurso de méritos. El 31 de octubre de 1933 finalizó sus estudios e inmediatamente inició su tesis de grado, la cual denominó "Ascaridiosis intestinal, sus complicaciones y tratamiento por el aceite esencial de quenopodio".

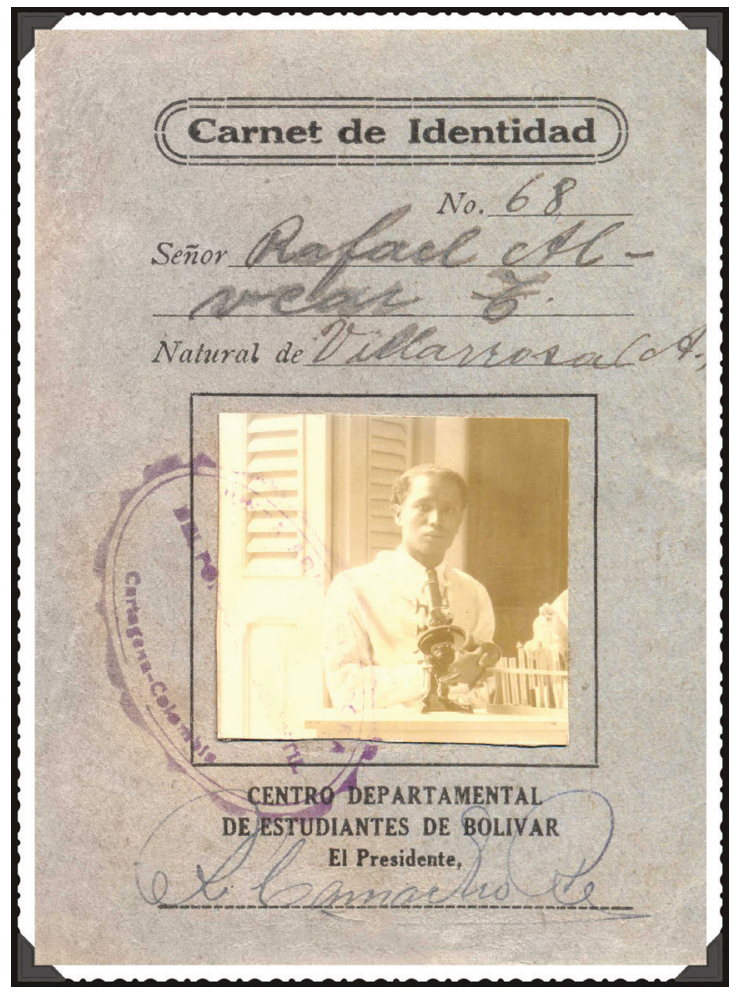

Facsímil del carné como integrante del Centro Departamental de Estudiantes de Bolívar.

El 1 de noviembre de 1935 obtuvo el título de Médico y Cirujano. La prensa local al respecto resaltó: "su juventud, sus capacidades, su especial don de gentes, su consagración al trabajo".

A la vez que cumplía con sus deberes inicialmente como estudiante y luego como médico, Alvear Terán incursionó por muchos años en la política local, regional y nacional. Fue por muchos años Concejal de Repelón, Diputado a la Asamblea del Atlántico, Representante a la Cámara por el departamento del Atlántico y Diputado a la Asamblea por el departamento de Bolívar. El doctor Alvear fue 


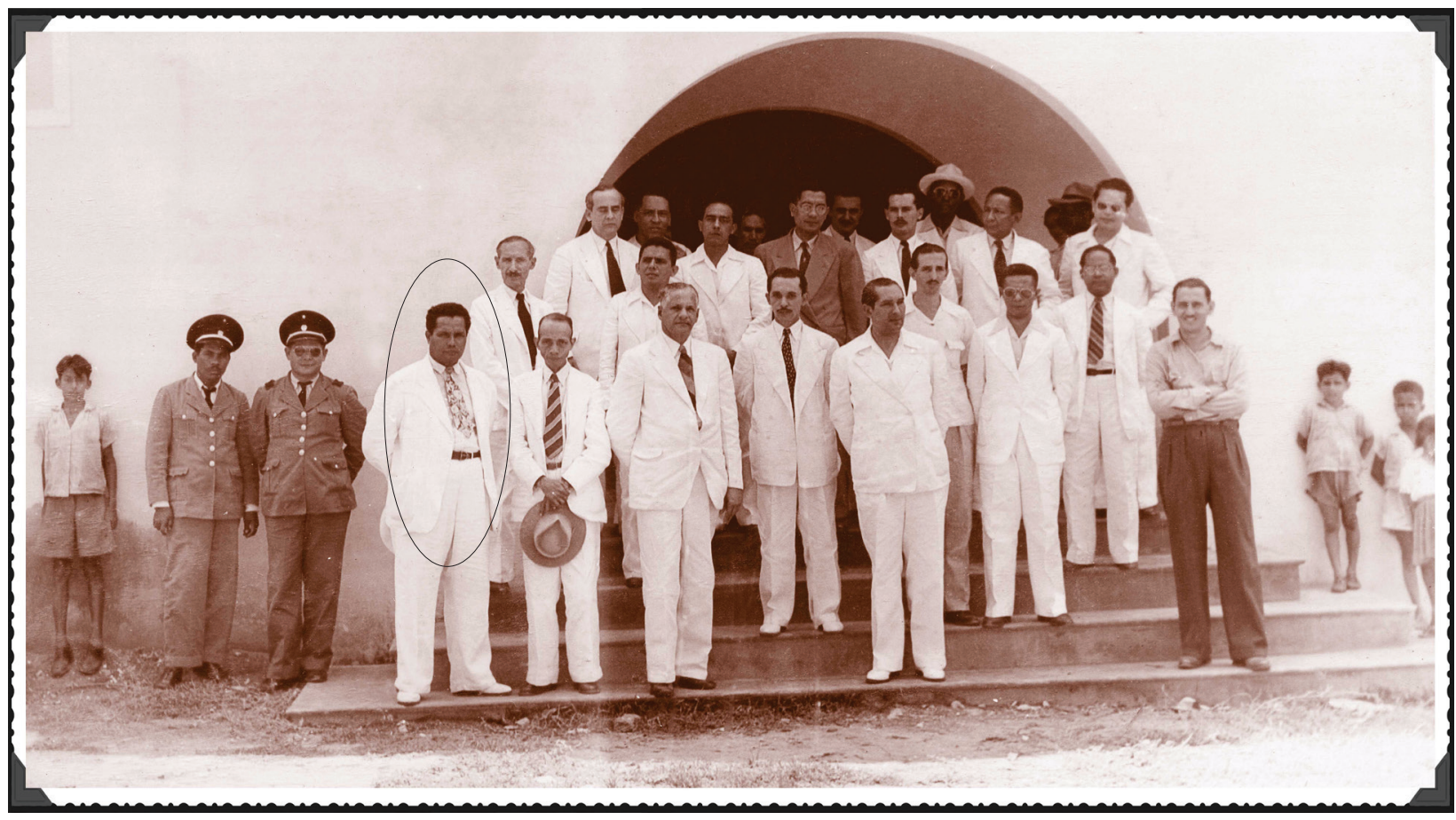

Rafael Alvear Terán junto a otros profesores de la Facultad de Medicina de la Universidad de Cartagena. A la puerta, al parecer de la Clínica de Maternidad Rafael Calvo en la quinta década del siglo XX.

un personaje admirado por sus compañeros políticos debido a su brillo, lucidez y elocuencia, siempre buscando obtener el progreso y el bienestar de la región. Es recordado aún como un hombre corpulento, de andar pausado, bien vestido, siempre de blanco como era la usanza de la época y siempre acompañado por un sombrero blanco.

De igual manera, era diestro en el conocimiento y la enseñanza de la anatomía humana, sobresalía en el arte literario por su creatividad. Escribió varios discursos y poesías que fueron compiladas en el libro: "Escasos frutos de mi erial". Fue miembro principal de la Junta Reformadora del Hospital Santa Clara, director por muchos años del Anfiteatro Anatómico de la Facultad de Medicina, Inspector de Sanidad (inspector médico) y ejerció el cargo de mé- dico de la Universidad para el cuerpo estudiantil, docente y administrativo por muchos años.

Además de la familia, la orden masónica a la cual pertenecía y la medicina, un importante pilar de su vida fue la anatomía. Rafael Alvear Terán ingresó como docente a la Facultad de Medicina de la Universidad de

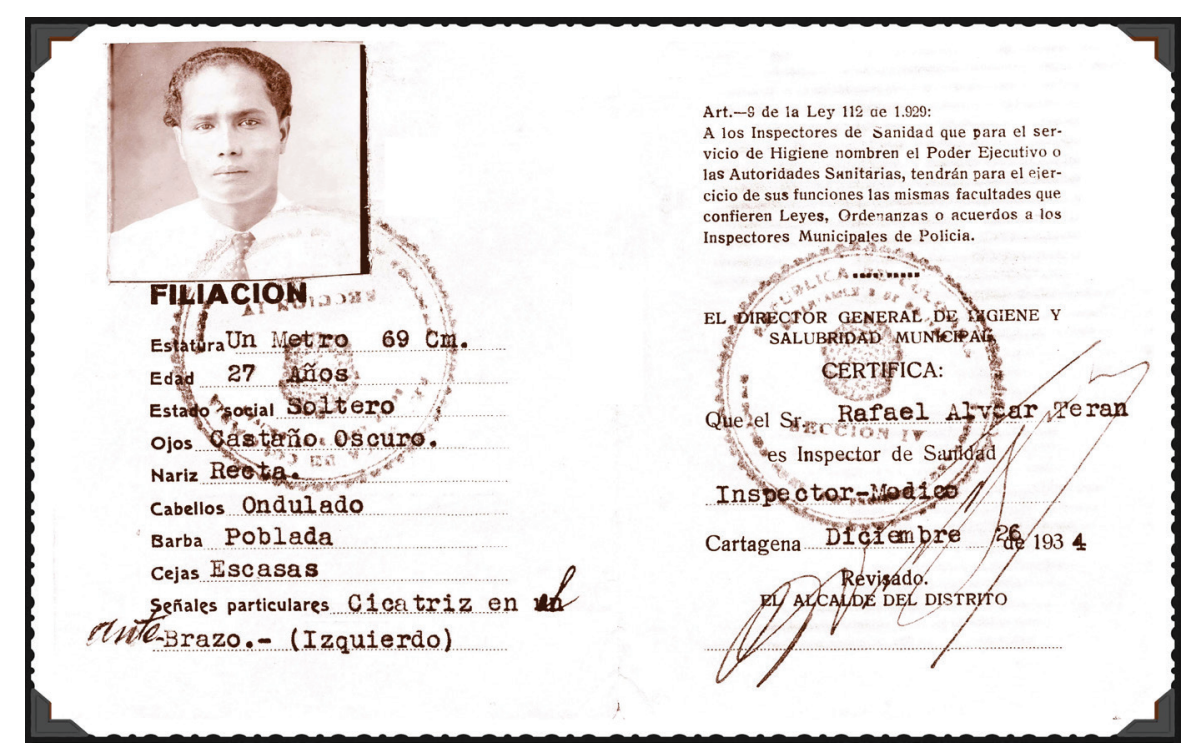

Facsímil del carné Inspector de Sanidad (inspector médico) 
Cartagena en 1939 como profesor interino de clínica terapéutica. En 1941 pasó a ser profesor interino de anatomía. De 1947 hasta 1951 fue profesor de biología humana. En 1953 estuvo vinculado como profesor interino de anatomía y desde 1955 fue profesor de anatomía. Por medio de la Resolución $N^{\circ}$ 90 del 31 de marzo de 1962, emanada de la Rectoría de la Universidad de Cartagena, fue clasificado como profesor de anatomía con el carácter de titular.

Realizó una recopilación en lo relacionado a las articulaciones y construyó un cuadro sinóptico acerca de los huesos del cuerpo humano. Ambos trabajos fueron adoptados para la enseñanza de la anatomía en la $\mathrm{Fa}-$ cultad de Medicina por muchos años. Estuvo al frente de su cátedra ininterrumpidamente hasta la jubilación en el año 1972. Fue el último docente, reconocido incluso por sus compañeros y profesores como anatomista, definido por los estrictos parámetros de un modelo que había ido desapareciendo del ámbito médico universal y reemplazado por otras corrientes, llenas de tecnologías diagnósticas y terapéuticas.

En sesión solemne del 19 de diciembre de 1985, Rafael Alvear Terán recibió del rector de la Universidad de Cartagena de ese entonces, Luis H. Arraut Esquivel y de manos del gobernador de Bolívar, Arturo Matson Figueroa, el diploma de Profesor Honorario, distinción otorgada por el Consejo Académico de la Universidad de Cartagena en homenaje a sus bodas de oro profesionales, al ejercicio que desempeñó con dedicación y competencia como Profesor Titular de la Cátedra de Anatomía y por su voluntad de servicio a la comunidad. La Cámara de Representantes de Colombia le confirió por Resolución No 131 del 7 de julio de 1995 la condecoración Orden de la Democracia en el grado de "Gran Cruz", por su aporte al engrandecimiento de la profesión médica.

Falleció en la ciudad de Cartagena-Colombia, el 12 de julio de 2002 a los 95 años de edad, en una época en que en la Facultad de Medicina de la Universidad de Cartagena siguiendo corrientes de educación médica universal, la cátedra de anatomía humana se había reducido notoriamente dentro del

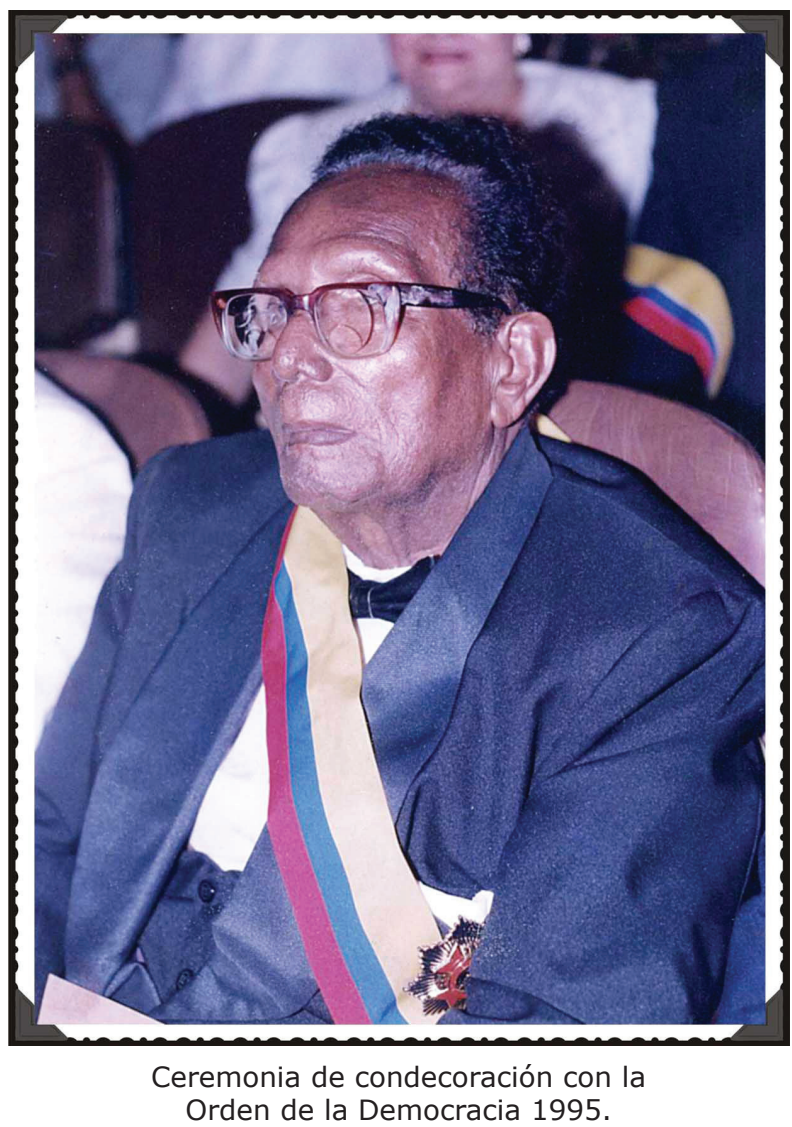

currículo, lo cual simplificó la magnitud de la necesidad del aprendizaje anatómico. Fueron cambiados los voluminosos tratados de anatomía por delgados y sencillos resúmenes. Era normal la casi o total desaparición de la necesidad de disección de cadáveres en el anfiteatro. El esplendor y el sitial de poder que tenía el anatomista dentro de la educación médica, estaba en el pasado.

\section{COLOFÓN}

La medicina anatomoclínica, desde la perspectiva médica actual, contenía considerables limitaciones, una de las más importantes es que se basaba en la observación, por lo tanto, era incapaz de reproducir los fenómenos en el laboratorio. Además de ello, no era posible conocer los agentes etiológicos de las enfermedades o explicar los mecanismos de desarrollo de los fenómenos morbosos. Ante esa debilidad, surgieron otros dos modelos para explicar la presencia de enfermedad y que también se pueden identificar dentro de la pedagogía médica. 
[A] MODELO FISIOPATOLÓGICO: se fundamenta en la experiencia obtenida dentro del laboratorio con el estudio del tejido o del ser vivo. Este modelo se observa en los planes curriculares de la educación médica colombiana en la mitad del siglo XX y entró en gran medida a reemplazar espacios educativos que utilizaba la enseñanza de la anatomía.

[B] MODELO ETIOPATOLÓGICO: explica la causa de las enfermedades por la acción de agentes externos y de microorganismos, modelo que también se incorporó rápidamente a la educación médica colombiana influenciada por las propuestas de Louis Pasteur (1822-1895). Estas procedían de los libros franceses y del conocimiento que trajeron médicos colombianos que realizaron estudios médicos o perfeccionamiento de sus conocimientos en hospitales franceses.

En 1948, Estados Unidos envió una Misión Médica a Colombia con el objeto de discutir e intervenir la enseñanza de la medicina en este país. Consecuentemente, en 1950 se creó el nuevo currículo, igual para todas las universidades del país, conforme a las recomendaciones dadas por la entidad norteamericanas basadas en el modelo de Flexner (1910) que promulgaba el desarrollo de una medicina de laboratorio basada en las concepciones fisiopatológicas y etiopatológicas, la construcción de los cimientos para una investigación básica y de adelantos sólidos en la tecnología médica.

Con el nuevo plan de estudios se le restó importancia a la enseñanza de la anatomía disminuyendo su alta intensidad horaria, se separaron las materias básicas de las clíni- cas y se plasmó la predominancia de la enseñanza práctica sobre la teórica. Las clases magistrales fueron criticadas y en su lugar se recomendó el trabajo práctico en el laboratorio, el hospital y la biblioteca. Son los planteamientos de la fisiopatología de Claude Bernard los que se tornan el centro o la rama dominante del saber médico. De esta manera, en Colombia la educación médica y el ejercicio de la medicina de corte francés comenzaron a ser desplazadas por una nueva propuesta, la medicina norteamericana.

Pedro Laín Entralgo (1908-2001), a las puertas del siglo XXI, analizó el modelo anatomoclínico y propuso el concepto de mentalidad anatomoclínica, donde involucró todo el fenómeno del cual la anatomía fue el epicentro de la enseñanza y asistencia médica. Dicho autor aseveró que la mentalidad anatomoclínica identifica en mayor medida a un fenómeno cultural y no solo, a un hecho aislado en la historia de la medicina universal. Esa postura permite observar coherentemente al acto médico desde el diagnóstico hasta la terapéutica y sirve de sustento para lo que Pierre Bordieu denominó "un habitus". Incluso Laín Entralgo, en su concepto de mentalidad anatomoclínica reconoce que este método, el fisiopatológico y el etiopatológico son valiosas conceptualizaciones o herramientas teóricas adecuadas para el estudio de la medicina universitaria del siglo XIX e incluso del siglo XX.

CONFLICTO DE INTERESES: ninguno que declarar.

FINANCIACIÓN: recursos propios de los autores.

\section{LECTURAS RECOMENDADAS}

1. Echeverri TÁ. Influencia francesa en la formación de la medicina antioqueña. Iatreia. $1994 ; 7(3): 148-53$.

2. Flexner A. Medical education. A comparative Study. Nueva York: The MacMillan Company; 1925.

3. García Usta J. Retratos de médicos. Crónicas sobre médicos del Bolívar Grande en el siglo XX. El pensamiento médico, selección de textos médicos (1890-1940). Bogotá: Editorial Gobernación de Bolívar; 2000.

4. Ghisays Ganem MA. Historia de la medicina en Cartagena de Indias. Editorial Universitaria. Universidad de Cartagena. 2016.

5. Hadju S. A note from history: The first histopathologists. Ann Clin Lab Sci. 2004; 34: 113315.

6. Hernández DPD. Una visión sucinta de la enseñanza de la medicina a través de la historia: II. Colombia, un sitio donde confluyeron varias culturas con su arte de curar. Iatreia. 2011; 24(2):207-14. 
7. Ligardo Vega R. Reconstrucción de un pasado médico: La Facultad de Medicina de la Universidad de Cartagena 1920-1940, entre el apostolado, la ciencia y el higienismo. La cátedra historia de la Universidad de Cartagena: Mirar su historia para construir su futuro. Cartagena de Indias: Editorial Universitaria; 2006.

8. Maza Anaya E. Facultad de Medicina de la Universidad de Cartagena Su historia. Cartagena: Editorial Universidad de Cartagena; 1998.

9. Miranda Canal N. La medicina en Colombia: de la influencia francesa a la norteamericana. Revista Credencial Historia. 1992.

10. Monterrosa-Castro A. Historia para conocer y recordar. La enseñanza-aprendizaje de la ginecología y obstetrícia en la Facultad de Medicina de la Universidad de Cartagena (18802009). Función Creativa. Bucaramanga. 2009.

11. Monterrosa-Castro A. La Gaceta Médica de la Sociedad de Medicina y Ciencias Naturales de Bolivar (1888-1919). Alpha Editores. 2015.

12. Pérez Perales JE. Marie-François Xavier Bichat y el nacimiento del método anatomoclínico. Cirujano general. 2011; 33(1):54-7.

13. Quevedo E, Pérez G, Miranda N, Eslava J, Hernández M, Bustos L, et al. Historia de la medicina en Colombia. Prácticas médicas en conflicto (1492-1782) Tomo I. Santander de Quilichao (Colombia): Norma; 2007

14. Romero Beltrán A. Historia de la medicina Colombiana: Siglo XIX. Medellín: Universidad de Antioquia; 1996.p.65.

15. Shoja M, Tubbs R, Loukas M, Shokouhi G, Ardalan M. Marie-Francois Xavier Bichat (17711802 ) and his contributions to the foundations of pathological anatomy and modern medicine. Ann Anat 2008; 190: 413-420.

16. Sillau Gilone J. Historia de la anatomía. Revista de la Sociedad Peruana de Neumología. 2005; 49.

17. Zabaleta Jaspe H. Réquiem por un viejo hospital. Bogotá: Ediciones Tercer Mundo Editores; 1976.p.173-179.

REVISTA CIENCIAS BIOMÉDICAS
Es el órgano de información científica de la Facultad de Medicina de la Universidad de Cartagena. Colombia.

Publique su trabajo en esta revista enviando su manuscrito a: revistacienciasbiomedicas@unicartagena.edu.co www.revistacienciasbiomedicas.com www.revistacienciasbiomedicas.com.co

Revista ciencias Biomédicas es una publicación independiente, imparcial, abierta, revisada por pares, de elevada visibilidad internacional, con circulación online e impresa. Publica artículos en todas las modalidades universalment aceptadas en inglés y en español, de temas referentes con todas las ciencias biomédicas, incluyendo ámbitos clínicos, epidemiológicos o de estudios básicos.

El sistema de gestión de manuscritos es rápido y justo.

Revista Ciencias Biomédicas está incluida en varias bases de datos latinoamericanas e internacionales.

Antes de enviar su manuscrito, revise las recomendaciones para los autores, presentes en: www.revistacienciasbiomedicas.com www.revistacienciasbiomedicas.com.co 